\title{
MATHEMATICAL MODEL OF THE MALE URINARY TRACT
}

\author{
Jiří Křen ${ }^{\mathrm{a}}$, Miroslav Horák ${ }^{\mathrm{a}}$, František Zátura ${ }^{\mathrm{b}}$, Míta Rosenberg $^{\mathrm{c}}$
}

a Department of Mechanics, University of West Bohemia, 30614 Plzeň, e-mail: kren@kme.zcu.cz
b Department of Urology, Faculty Hospital, Palacký University, 725 20 Olomouc, e-mail: zaturaf@fnol.cz
c Special physician for urology-private practice, Kaznějovská 45, 32300 Plzeñ, Czech Republic

Received: November 9, 2001

Keywords: Interaction / Uncoupled method / Non-linear continuum / Equation of motion / FEM

The paper presents a simplified (but not trivial) mathematical model of the interaction between the urine flow and the male urethra and bladder, respectively. Urine is assumed to be a Newtonian fluid. The flow is considered to be non-stationary, isothermal and turbulent. The urethra and bladder wall, featuring elastic properties, experience large displacements and strains. The dynamic forces are included in the urethra wall motion. When fully extended the urethra attains the shape of an axisymetric tube. An iterative method based on the uncoupled approach is developed.

\section{INTRODUCTION}

Constituting a mathematical model of the urine flow in a male urethra and bladder is one of the goals of the research grant GA ČR, which is solved at the Department of Mechanics of the UWB, Pilsen with the cooperation of Department of Urology, Palacký University, and M. Rosenberg, special physician for urology-private practice. The solution represents a very complicated problem of interaction of fluid with deformable body with a time dependent position of the interacting continuums boundary (free boundary problem).

Basic characteristics of a real lower part of the urinary tract: the urine from the bladder flows through a cylindrical non-rotary tube (urethra) with a flexible rough wall, the urine flow is non-stationary (pressure change in the bladder), the flow is not fully developed in the parts of the bladder neck and along the most part of the urethra is turbulent; the urethra and bladder wall tissue are a non-isotropic and generally non-linear viscoelastic material; the urethra is in a resting state a deflated tube, which is first opened under the urine pressure and only afterwards is stretched.

Regarding the complexity of the solved problem we limited ourselves, in the first approach, to the solution of an "elastic" model problem of interaction of the urine with the flexible urethra and bladder wall. The following assumptions are accepted: micturation process is fully developed, i. e. the bladder has a shape of an ellipsoid and the urethra has a shape of a rotary cylindrical tube with variable cross-section, the urine is a Newtonian fluid, its flow is non-stationary, turbulent and isothermal; the urethra and bladder wall material are an isotropic linear elastic ones with known constitutive relation; the urethra and bladder tissue are subjected to large displacements and deformations, i. e. urethra and bladder deformation solution represents a geometrically nonlinear problem.
To solve the above model problem we use an uncoupled approach to the interaction problem solution, which is based on the assumption of the independent solution of the motion of both the interacting continua considering the fact that at the common boundary (interaction boundary) the continua influence each other. Then, the interaction problem is divided to an independent solution of the urine flow in a deformable bladder and "rigid" urethra and to the solution of the urethra wall deformation itself.

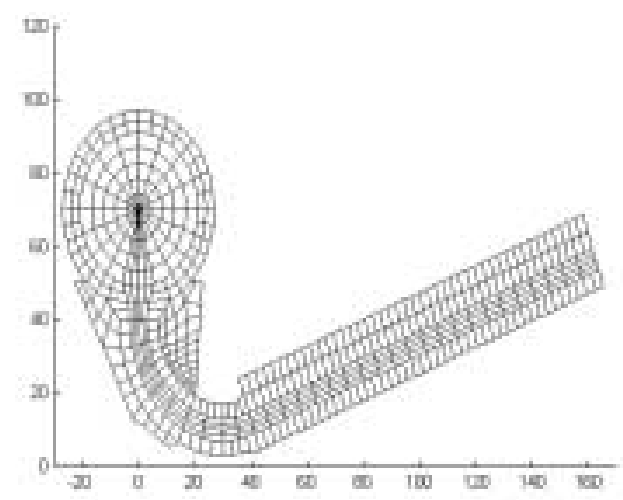

Velocity distribution in the urethra
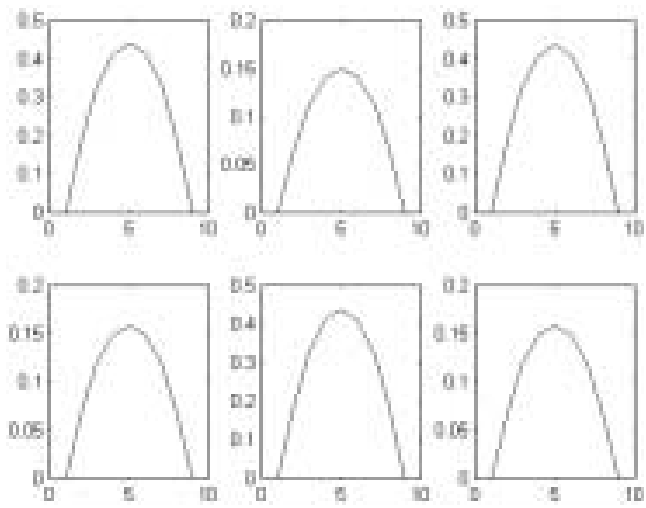

Fig. 1 Starting computer model of the male urinary tract (plane model) 
Non-stationary flow of newtonian fluid through bladder and urethra

The boundary and initial problem of hydromechanics is generally described by the well-known Navier-Stokes equation, continuity equation and respective boundary conditions (e. g. form. (1)). Original equations for laminar flow of a Newtonian fluid have a form (components of the velocity vector $v_{j}^{*}$ are regarded as known ones)

$\rho \frac{\partial v_{i}}{\partial t}+\rho v_{j}^{*} \frac{\partial v_{i}}{\partial x_{j}}=-\frac{\partial p}{\partial x_{i}}+\mu \frac{\partial}{\partial x_{j}}\left(\frac{\partial v_{i}}{\partial x_{j}}\right)+\rho f_{i} ;$

$\frac{\partial v_{i}}{\partial x_{i}}=0 ; \quad t \in(0, T), x \in \Omega$

Boundary and initial conditions of the flow have a form $v_{i}(x, t)=\widehat{v}_{i}(x, t) ; t \in(0, T), x \in \partial \Omega_{1}$,

$\tau_{i j} n_{j}=\widehat{\sigma}_{i}(x, t) ; t \in(0, T), x \in \partial \Omega$,

$v_{i}(x, 0)={ }^{0} \widehat{v}_{i}(x) ; \quad t=0, x \in \Omega$.

Here $\Omega$ is a domain with the Lipschitz's boundary $\partial \Omega=\partial \Omega_{1} \cup \partial \Omega_{2}, \bar{\Omega}=\Omega \cup \partial \Omega, x_{i}$ are Euler's co-ordinates, $v_{\mathrm{i}}$ are components of the velocity vector, $p$ is pressure, $\mu$ is dynamic viscosity of the Newtonian fluid, $p$ is density and $f_{i}$ are components of the specific volume force. Unknowns in this hydromechanic problem are $\left\{v_{\mathrm{v}}, p\right\}$. The solution will be search on the time-spatial cylinder $\bar{D}=\bar{\Omega} \times[0, T]$.

Using Galerkin's method for deriving weak solution of this hydromechanic problem the initial integral relations have a form

$$
\begin{aligned}
& \int_{\Omega} \rho \frac{\partial v_{i}}{\partial t} \delta v_{i} d x+\int_{\Omega} \rho v_{j}^{*} \frac{\partial v_{i}}{\partial x_{j}} \delta v_{i} d x-\int_{\Omega} p \frac{\partial \delta v_{i}}{\partial x_{i}} d x+ \\
& +\int_{\Omega} \mu \frac{\partial v_{i}}{\partial x_{j}} \frac{\partial \delta v_{i}}{\partial x_{j}} d x=\int_{\Omega} \rho f_{i} \delta v_{i} d x-\int_{\tilde{\Omega} \Omega_{2}}\left(\mu \frac{\partial v_{i}}{\partial \vec{n}}-p n_{i}\right) \delta v_{i} d x ; \\
& \int_{\Omega} \frac{\partial v_{i}}{\partial x_{i}} \delta p d x=0 .
\end{aligned}
$$

Let us perform a spatial discretization of the domain filled by the fluid using FEM. Using isoparametric interpolation functions the velocity components $\left(v_{1}=u, v_{2}=v, v_{3}=w\right)$ and pressure $p$ can be approximated as

$$
\begin{aligned}
& u(\xi, \eta, \zeta, t)=\boldsymbol{N}^{T}(\xi, \eta, \zeta) \boldsymbol{u}(t) \\
& v(\xi, \eta, \zeta, t)=\boldsymbol{N}^{T}(\xi, \eta, \zeta) \boldsymbol{v}(t) \\
& w(\xi, \eta, \zeta, t)=\boldsymbol{N}^{T}(\xi, \eta, \zeta) \boldsymbol{w}(\boldsymbol{t}) \\
& p(\xi, \eta, \zeta, t)=\boldsymbol{H}^{T}(\xi, \eta, \zeta) \boldsymbol{p}(t)
\end{aligned}
$$

where $\boldsymbol{N}$ and $\boldsymbol{H}$ are vectors of interpolation functions defined locally for each element and $\boldsymbol{u}, \boldsymbol{v}, \boldsymbol{w}, \boldsymbol{p}$ are vectors of nodal quantities of velocity and pressure components. From relations (4), (5) and (6) four equations in a matrix form ( $\mu=$ const,$\rho=$ const $)$ can be obtained

$$
\begin{aligned}
& A_{1} \dot{u}+B_{1}\left(u^{*}, v^{*}, w^{*}\right) u+D_{1} u+C_{1} p=E_{1}, \\
& A_{1} \dot{v}+B_{1}\left(u^{*}, v^{*}, w^{*}\right) v+D_{1} v+C_{2} p=E_{2}, \\
& A_{1} w+B_{1}\left(u^{*}, v^{*}, w^{*}\right) w+D_{1} v+C_{3} p=E_{3}, \\
& K_{1} u+K_{2} v+K_{3} w=0
\end{aligned}
$$

Expressing the velocity time change using a difference scheme

$\dot{\boldsymbol{u}}=\frac{\boldsymbol{u}^{n+1}-\boldsymbol{u}^{n}}{\Delta t} ; \quad \dot{\boldsymbol{v}}=\frac{\boldsymbol{v}^{n+1}-\boldsymbol{v}^{n}}{\Delta t} ; \quad \dot{\boldsymbol{w}}=\frac{\boldsymbol{w}^{n+1}-\boldsymbol{w}^{n}}{\Delta t}$,

the above equation system (7) can be rewritten in a compact matrix form

$$
\left[\begin{array}{cccc}
\boldsymbol{A} & \boldsymbol{0} & \boldsymbol{0} & \boldsymbol{B} \\
\boldsymbol{0} & \boldsymbol{A} & \boldsymbol{0} & \boldsymbol{C} \\
\boldsymbol{0} & \boldsymbol{0} & \boldsymbol{A} & \boldsymbol{D} \\
\boldsymbol{K} & \boldsymbol{K} & \boldsymbol{K} & \boldsymbol{0}
\end{array}\right]\left[\begin{array}{c}
\boldsymbol{u} \\
\boldsymbol{v} \\
\boldsymbol{w} \\
\boldsymbol{p}
\end{array}\right]^{(n+1)}=\Delta t\left[\begin{array}{c}
\boldsymbol{E}_{1} \\
\boldsymbol{E}_{2} \\
\boldsymbol{E}_{3} \\
\boldsymbol{0}
\end{array}\right]^{(n+1)}+\left[\begin{array}{c}
\boldsymbol{F}_{1} \\
\boldsymbol{F}_{2} \\
\boldsymbol{F}_{3} \\
\boldsymbol{0}
\end{array}\right]^{(n)},
$$

where newly included matrices have generally a form $\mathbf{A}=\mathbf{A}_{1}+\Delta t(1-\beta)\left|\mathbf{B}_{1}\left(\mathbf{u}^{*}, \mathbf{v}^{*}, \mathbf{w}^{*}\right)^{(n+1)}+\mathbf{D}_{1}\right|, \mathbf{B}=\Delta t(1-\beta) \mathbf{C}_{1}, \mathbf{C}=\Delta t(1-\beta) \mathbf{C}_{2}$ $\mathbf{F}_{1}=\left\{\mathbf{A}_{1}-\Delta t \beta\left[\mathbf{B}_{1}\left(\mathbf{u}^{*}, \mathbf{v}^{*}, \mathbf{w}^{*}\right)^{(n)}+\mathbf{D}_{1}\right] \boldsymbol{\beta}^{(n)}-\Delta t \boldsymbol{B} \mathbf{C} \mathbf{p}^{(n)}, \mathbf{D}=\Delta t(1-\beta) \mathbf{C}_{3}\right.$,

$\mathbf{F}_{2}=\left\{\mathbf{A}_{1}-\Delta t \beta\left[\mathbf{B}_{1}\left(\mathbf{u}^{*}, \mathbf{v}^{*}\right)^{(n)}+\mathbf{D}_{1}\right\}\right\}^{(n)}-\Delta t \beta \mathbf{C}_{2} \mathbf{P}^{(n)}$,

$\left.\mathbf{F}_{3}=\left\{\mathbf{A}_{1}-\Delta t \beta \mid \mathbf{B}_{1}\left(\mathbf{u}^{*}, \mathbf{v}^{*}, \mathbf{w}^{*}\right)^{(n)}+\mathbf{D}_{1}\right\}\right\}_{\mathbf{w}^{(n)}}-\Delta t \beta \mathbf{C}_{3} \mathbf{p}^{(n)}$.

Coefficient $\beta$ can has following quantities (explicit or implicit solution scheme)

$\beta=\left\{\begin{array}{lll}0 & \text { for } & \boldsymbol{u}=\boldsymbol{u}^{(n+1)}, \boldsymbol{v}=\boldsymbol{v}^{(n+1)}, \boldsymbol{p}=\boldsymbol{p}^{(n+1)} \\ \frac{1}{1} & \text { for } & \boldsymbol{u}=\frac{\boldsymbol{u}^{(n+1)}+\boldsymbol{u}^{(n)}}{2}, \boldsymbol{v}=\frac{\boldsymbol{v}^{(n+1)}+\boldsymbol{v}^{(n)}}{2} \\ \frac{\boldsymbol{2}}{2} & & \boldsymbol{p}=\frac{\boldsymbol{p}^{(n+1)}+\boldsymbol{p}^{(n)}}{2} \\ 1 & \text { for } & \boldsymbol{u}=\boldsymbol{u}^{(n)}, \boldsymbol{v}=\boldsymbol{v}^{(n)}, \boldsymbol{p}=\boldsymbol{p}^{(n)}\end{array}\right.$

where $n$ is the time level. The matrix form (9) represents a system of non-linear algebraic equations, which is solved by Newton-Raphson's method at each time level. In shortened record of the system of equations (9) in the form ( $x$ represents a vector of unknowns of the flow problem)

$\boldsymbol{K}^{(n+1)} \boldsymbol{x}^{(n+1)}=\boldsymbol{f}^{(n)}$,

the iterative computational process is controlled by relations ( $n$-time level, $r$-iterative step inside the actual time level) 


$$
\begin{aligned}
& \boldsymbol{x}^{(n+1),(r+1)}=\boldsymbol{x}^{(n+1),(r)}-\boldsymbol{J}_{(n+1),(r)}^{-1} \boldsymbol{R}^{(n+1),(r) ;(n),(0)} ; \\
& \boldsymbol{R}^{(n+1),(r) ;(n),(0)}=\boldsymbol{K}^{(n+1),(r)} \boldsymbol{x}^{(n+1),(r)}-\boldsymbol{f}^{(n),(0)} .
\end{aligned}
$$

It means that velocities and pressures in the $f^{(\mathrm{n})}$ vector are "constants" calculated in the previous time level and they do not change during the iterative process at the actual time level. $\boldsymbol{J}$ is the Jacobian matrix [5]. By this the problem of a non-stationary laminar flow of a Newtonian fluid in urethra is solved.

Let us consider now a turbulent flow of a Newtonian fluid. Let us suppose that velocity components $v_{i}$ and pressure $p$ in relations (1) and (2) can be expressed in a form

$$
v_{i}=\bar{v}_{i}+v_{i}^{\prime} ; \quad p=\bar{p}+p^{\prime},
$$

where $\bar{v}_{i}, \bar{p}$ are time average quantities and $v_{i}^{\prime}, p^{\prime}$ are fluctuating quantities. We will now proceed to establish the equations (1), (2) in terms of time average quantities. The second term of the Navier-Stokes equation can be expressed in a form (by means of the continuity equation)

$\rho v_{j} \frac{\partial v_{i}}{\partial x_{j}}=\rho \frac{\partial\left(v_{j} v_{i}\right)}{\partial x_{j}}$, resp. $\rho \frac{\partial\left(\overline{v_{j} v_{i}}\right)}{\partial x_{j}}=\rho \bar{v}_{j} \frac{\partial \bar{v}_{i}}{\partial x_{j}}+\rho \frac{\partial\left(\overline{v_{j}^{\prime} v_{i}^{\prime}}\right)}{\partial x_{j}}$

Let us substitute equation (14) and (15) into equations (1) and (2). We obtain $\left(\overline{p^{\prime}}=0, \overline{v_{i}^{\prime}}=0\right.$; with the turbulence term on the right side of the Naveir-Stokes equation)

$$
\begin{aligned}
& \rho \frac{\partial \bar{v}_{i}}{\partial t}+\rho \bar{v}_{j} \frac{\partial \bar{v}_{i}}{\partial x_{j}}=-\frac{\partial \bar{p}}{\partial x_{i}}+\frac{\partial}{\partial x_{j}}\left(\mu \frac{\partial \bar{v}_{i}}{\partial x_{j}}-\rho \overline{v_{j}^{\prime} v_{i}^{\prime}}\right)+\rho \bar{f}_{i} \\
& \frac{\partial \bar{v}_{i}}{\partial x_{i}}=0 .
\end{aligned}
$$

These equations are equations for turbulent flow written in terms of time average quantities. First equation differs from the equation for instantaneous quantities, equation (1), only by the addition of the last term (the shear stress tensor $=$ laminar $\tau_{i j}^{L}$ plus turbulent $\left.\tau_{i j}^{T}\right)$. This term is often referred to as the Reynolds stresses or turbulent stresses. It can be expressed in a form [1]

$$
\tau_{i j}^{T}=-\rho \overline{v_{j}^{\prime} v_{i}^{\prime}}=\rho \varepsilon_{\tau} \frac{\partial \bar{v}_{i}}{\partial x_{j}}=\mu_{T} \frac{\partial \bar{v}_{i}}{\partial x_{j}} ; \text { for } i \neq j,
$$

where $\mu_{T}$ is turbulent viscosity and $\varepsilon_{\tau}$ is referred to as the eddy viscosity. We will assume that $\mu_{T}=$ const. The equations (16), (17) can be then rewritten in a form

$$
\begin{aligned}
& \rho \frac{\partial \bar{v}_{i}}{\partial t}+\rho \bar{v}_{j}^{*} \frac{\partial \bar{v}_{i}}{\partial x_{j}}=-\frac{\partial \bar{p}}{\partial x_{i}}+\left(\mu+\mu_{T}^{*}\right) \frac{\partial}{\partial x_{j}}\left(\frac{\partial \bar{v}_{i}}{\partial x_{j}}\right)+\overline{\rho f_{i}} \\
& \frac{\partial \bar{v}_{i}}{\partial x_{i}}=0 .
\end{aligned}
$$

These equations are formally the same as the basic equations (1) and (2). Thus for the numerical solution above algorithms (3) to (15) are valid. Notice that turbulent viscosity $\mu_{T}^{*}$ can be determined by means of e. $g$. Prandtl mixing length theory. The problem of the urine turbulent flow in the urethra is thus solved.

\section{Bladder and urethra wall deformation}

Let us consider a motion of a flexible body in a fixed Carthesian co-ordinate system. The body (continuum) performs large generalised displacements and produces large deformations. The goal of the solution is to determine equilibrium positions of continuum in discrete time points $0, \Delta t, 2 \Delta t, \ldots, t, t+\Delta t, \ldots, T$, where $\Delta t$ is a time increment and all changes of continuum during its motion are supposed to be continuous. A transition of continuum from the $t\left({ }^{t} C\right)$ - time configuration to that of $t+\Delta t\left({ }^{t+\Delta t} C\right)$ one is thus a typical step of the mechanics of continuum non-linear problem solution, which is usually performed by an iterative method. In the solution we consider the motion of all continuum particles, i. e. we apply the Lagrange's description of continuum and we use an incremental method.

The equilibrium position of continuum in $t+\Delta t$ time can be expressed using the virtual work principle [5]

$$
\int_{t+\Delta_{V}}^{t+\Delta t} \tau_{i j} \delta_{t+\Delta t} e_{i j} d^{t+\Delta t} V=\delta^{t+\Delta t} W
$$

where $\tau_{i j}$ is Cauchy's stress tensor, $e_{i j}$ is the linear part of Almansi's strain tensor and the virtual work of external forces is expressed as

$$
\delta^{t+\Delta t} W=\int_{t+\Delta t_{V}}^{t+\Delta t} f_{i}^{B} \delta u_{i} d^{t+\Delta t} V+\int_{t+\Delta_{S}}^{t+\Delta t} f_{i}^{S} \delta u_{i}^{S} d^{t+\Delta t} S .
$$

In these relations all the quantities are related to the instant continuum configuration ${ }^{t+\Delta t} C, f_{i}^{B}$ and $f_{i}^{S}$ are components of external body-forces and surface-forces. However, since the continuum state in $t+\Delta t$ time is not known, the formula (22) cannot be used for the calculation directly. That is why in solutions of continuum mechanics non-linear problem the incremental formulation of the virtual work principle is used, where all the continuum quantities are related to a certain known equilibrium state of continuum reference configuration.

Here we limit ourselves to the so-called total Lagrange's formulation (TL formulation), where the reference configuration is the continuum configuration ${ }^{\circ} \mathrm{C}$ (in $t=0$ time). The Lagrange's formulation is in general based on the identity (e. g. [2], [5])

$$
\int_{{ }_{0}}{ }_{0}^{t} S_{i j} \delta_{0}^{t} \varepsilon_{i j} d^{0} V=\int_{{ }^{t} V}^{t} \tau_{m n} \delta^{t} e_{m n} d^{t} V,
$$

which expresses the continuum internal force virtual work in respective configurations. ${ }_{0}^{t} S_{i j}$ and ${ }_{0}^{t} \varepsilon_{i j}$ are the second Piola-Kirchhoff's stress tensor and Green's strain 
tensor resp. in the configuration ${ }^{t} C$ related to (measured at) the configuration ${ }^{\circ} \mathrm{C}$. Green's strain tensor has a form $u_{i}(-$ displacements $)$

${ }_{0}^{t} \varepsilon_{i j}=\frac{1}{2}\left({ }_{0}^{t} u_{i, j}+{ }_{0}^{t} u_{j, i}+{ }_{0}^{t} u_{k, i 0}{ }^{t} u_{k, j}\right)$

${ }_{0}^{t} u_{i, j}=\frac{\partial^{t} u_{i}}{\partial^{0} x_{j}}$.

The TL formulation solution in thus conjugated to the ${ }^{\circ} \mathrm{C}$ configuration and its basic quantities have values:

- at the increment beginning (start point)

${ }^{t} u_{i},{ }_{0}^{t} \varepsilon_{i j},{ }_{0}^{t} S_{i j}$ (determine ${ }^{t} C$ configuration),

- at the increment end

$$
{ }^{t} u_{i}+\Delta u_{i},{ }_{0}^{t} \varepsilon_{i j}+\Delta_{0}^{t} \varepsilon_{i j},{ }_{0}^{t} S_{i j}+\Delta_{0}^{t} S_{i j}
$$

(determine ${ }^{t+\Delta t} C$ configuration).

The strain tensor increment $\Delta_{0}^{t} \varepsilon_{i j}$ has, according (24), two parts - a linear $\Delta_{0}^{t} \varepsilon_{i j}^{L}$ one and a non-linear $\Delta_{0}^{t} \varepsilon_{i j}^{N L}$ one, being (e.g. [3])

$$
\begin{aligned}
& \Delta_{0}^{t} \varepsilon_{i j}^{L}=\frac{1}{2}\left(\frac{\partial \Delta u_{i}}{\partial^{0} x_{j}}+\frac{\partial \Delta u_{j}}{\partial^{0} x_{i}}+\frac{\partial^{t} u_{k}}{\partial^{0} x_{i}} \frac{\partial \Delta u_{k}}{\partial^{0} x_{j}}+\frac{\partial \Delta u_{k}}{\partial^{0} x_{i}} \frac{\partial^{t} u_{k}}{\partial^{0} x_{j}}\right) \\
& \Delta_{0}^{t} \varepsilon_{i j}^{N L}=\frac{1}{2} \frac{\partial \Delta u_{k}}{\partial^{0} x_{i}} \cdot \frac{\partial \Delta u_{k}}{\partial^{0} x_{j}} .
\end{aligned}
$$

Substituting the above relations into the equation (23) and adopting approximations ${ }_{0} E_{i j k l}$ (is a material tensor related to ${ }^{\circ} \mathrm{C}$ )

$$
\Delta_{0}^{t} S_{i j}={ }_{0} E_{i j k l} \Delta_{0}^{t} \varepsilon_{k l}^{L} ; \quad \delta\left(\Delta_{0}^{t} \varepsilon_{i j}\right)=\delta\left(\Delta_{0}^{t} \varepsilon_{i j}^{L}\right)
$$

we obtain the basic form of continuum equilibrium condition in the incremental TL formulation, which we solve in the range of a given increment by an iterative method. There is [3]

$$
\begin{aligned}
& \int_{{ }^{0}} \delta\left(\Delta_{0}^{t} \varepsilon_{i j}^{L}\right)^{(\mathrm{k})}{ }_{0} E_{i j k l} \Delta_{0}^{t} \varepsilon_{k l}^{L(k)} d^{0} V+\int_{{ }_{V}} \delta\left(\Delta_{0}^{t} \varepsilon_{i j}^{N L}\right)^{(\mathrm{k})}{ }_{0}^{t} S_{i j} d^{0} V= \\
& =\delta^{t+\Delta t} W-\int_{{ }^{0}} \delta\left(\Delta_{0}^{t} \varepsilon_{i j}\right)^{(\mathrm{k}-1)}{ }_{0}^{t} S_{i j}^{(k-1)} d^{0} V \\
& { }^{t+\Delta t} u_{i}^{(k)}={ }^{t+\Delta t} u_{i}^{(k-1)}+\Delta u_{i}^{k} ; \quad{ }^{t+\Delta t} u_{i}^{(0)}={ }^{t} u_{i} .
\end{aligned}
$$

Notify, that for the solution of the equation (29) the modified Newton-Raphson's method is mostly used, which requires using the constant tangential stiffness matrix in each step.

Performing spatial discretization of continuum by FEM the relation (29) gives for each increment a matrix equation [3]

$$
\begin{aligned}
& {\left[\boldsymbol{K}_{1}+\boldsymbol{K}_{2}(\boldsymbol{q})+\boldsymbol{K}_{\sigma}\right] \Delta \boldsymbol{q}^{(k)}={ }^{t+\Delta t} \boldsymbol{Q}-{ }^{t+\Delta t} \boldsymbol{R}^{(k-1)}} \\
& \boldsymbol{K}_{\boldsymbol{t}} \Delta \boldsymbol{q}^{(k)}={ }^{t+\Delta t} \boldsymbol{Q}-{ }^{t+\Delta t} \boldsymbol{R}^{(k-1)},
\end{aligned}
$$

where the term in square brackets is a tangential stiffness matrix $\boldsymbol{K}_{\boldsymbol{t}}$, which consists of a linear stiffness matrix, an initial strains matrix and an initial stress matrix. $\boldsymbol{q}$ is a vector of generalised co-ordinates of continuum, $\boldsymbol{Q}$ is a vector of generalised forces and $\boldsymbol{R}$ is a vector of continuum internal forces (stress forces). Thus, the problem of the bladder and urethra wall deformation (elastic continuum) are solved.

\section{Dynamics of the bladder and urethra wall}

For the dynamic solution of the male bladder and urethra motion we need to introduce into equations (29) or (31) dynamic forces. We know that these forces are external body-forces and volume-forces, respectively. Thus we can express components of the external body force $f_{i}^{B}$ in the equation (22) by means of D'Alambert principle. The virtual work of the volume-forces for incompressible material can be written as

$$
\int_{t+\Delta t_{V}}^{t+\Delta t} \rho^{t+\Delta t} \ddot{u}_{i} \delta \dot{u}_{i}^{t+\Delta t} d V=\int_{0_{V}}^{0} \rho^{t+\Delta t} \ddot{u}_{i} \delta \dot{u}_{i}{ }^{0} d V
$$

because density $\rho$ of the tissues is time independent. From this equation yields practice conclusion that mass matrix for the isoparametric concept can be determined before the incremental method solution. It means that mass matrix is time independent.

Performing spatial discretization of continuum by FEM the relation (31) gives for each increment (with respect Eq. (33)) a matrix equation of continuum motion [4]

$$
\begin{aligned}
& \boldsymbol{M}^{t+\Delta t} \ddot{\boldsymbol{q}}^{(k)}+\boldsymbol{K}_{t} \Delta \boldsymbol{q}^{(k)}={ }^{t+\Delta t} \boldsymbol{Q}-{ }^{t+\Delta t} \boldsymbol{R}^{(k-1)} \\
& { }^{t+\Delta t} \boldsymbol{q}^{(k)}={ }^{t+\Delta t} \boldsymbol{q}^{(k-1)}+\Delta \boldsymbol{q}^{(k)}
\end{aligned}
$$

Equation (34) we solve in the range of a given increment by an iterative method, too (modified Newton-Raphson's method).

Now we need to express a vector ${ }^{t+\Delta t} \boldsymbol{q}$ and ${ }^{t+\Delta t} \ddot{\boldsymbol{q}}$, respectively, using generalised co-ordinates of continuum. Using the linear law for the time numerical integration we obtain relations

$$
\begin{aligned}
& { }^{t+\Delta t} \boldsymbol{q}={ }^{t} \boldsymbol{q}+\frac{\Delta t}{2}\left({ }^{t} \dot{\boldsymbol{q}}+{ }^{t+\Delta t} \dot{\boldsymbol{q}}\right), \\
& { }^{t+\Delta t} \dot{\boldsymbol{q}}={ }^{t} \dot{\boldsymbol{q}}+\frac{\Delta t}{2}\left({ }^{t} \ddot{\boldsymbol{q}}+{ }^{t+\Delta t} \ddot{\boldsymbol{q}}\right) .
\end{aligned}
$$

From equations (35) - (37) then yields

$$
{ }^{t+\Delta t} \ddot{\boldsymbol{q}}^{(k)}=\frac{4}{(\Delta t)^{2}}\left[{ }^{t+\Delta t} \boldsymbol{q}^{(k-1)}-{ }^{t} \boldsymbol{q}+\Delta \boldsymbol{q}^{(k)}\right]-\frac{4}{\Delta t}{ }^{t} \dot{\boldsymbol{q}}-{ }^{t} \ddot{\boldsymbol{q}}
$$

and equation (34) can be rewritten as

$$
\begin{aligned}
& \left.{ }^{t} \hat{\boldsymbol{K}} \Delta \boldsymbol{q}^{(k)}={ }^{t+\Delta} \boldsymbol{Q}-{ }^{t+\Delta} \boldsymbol{R}^{(k-1)}-\boldsymbol{M}\left\{\frac{4}{(\Delta t)^{2}}{ }^{t+\Delta t} \boldsymbol{q}^{(k-1)}-{ }^{t} \boldsymbol{q}\right]-\frac{4}{\Delta t}{ }^{t} \dot{\boldsymbol{q}}-{ }^{t} \ddot{\boldsymbol{q}}\right\} \\
& { }^{t} \widehat{\boldsymbol{K}}=\boldsymbol{K}_{t}+\frac{4}{(\Delta t)^{2}} \boldsymbol{M} .
\end{aligned}
$$


The form of this equation is formally the same as equation (32). It means that for the numerical solution of equation (40) we use the same modified NewtonRaphson's method as in the static deformation problem solution.

\section{Interaction problem solution}

With accordance to the uncoupled method of the interaction solution we use again an iterative method. First we solve the problem of bladder deformation (TL formulation, bladder is loaded by pressure $p$ ) and then the urine flow in a "rigid" urethra which yields to the urine pressure distribution along the urethra. By this pressure an elastic urethra is loaded and its deformation is solved using the incremental TL formulation. However, because of the urethra wall deformation, the area for the urine flow will change and thus the previous flow calculation has to be corrected. This iterative process takes place until the equilibrium position of both the interacting continua is found, i. e. until the prescribed accuracy conditions of the solution are fulfilled (the most sensitive quantity is the urine pressure). The solution is performed at the appropriate chosen time levels and inside every time level the above iterative method is applied. The solution requires using adaptive finite element mesh. The results obtained by the above algorithm are shown in the figures.

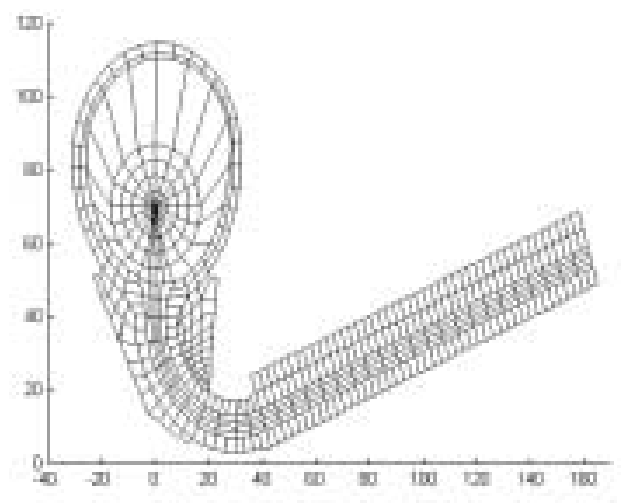

Velocity distribution in the urethra
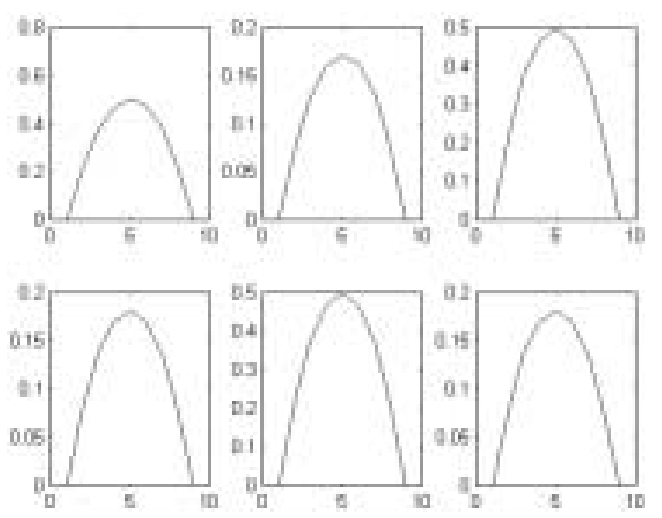

Fig. 2 Influence of the deformable bladder shape on the urine flow (plane model, "rigid" urethra)

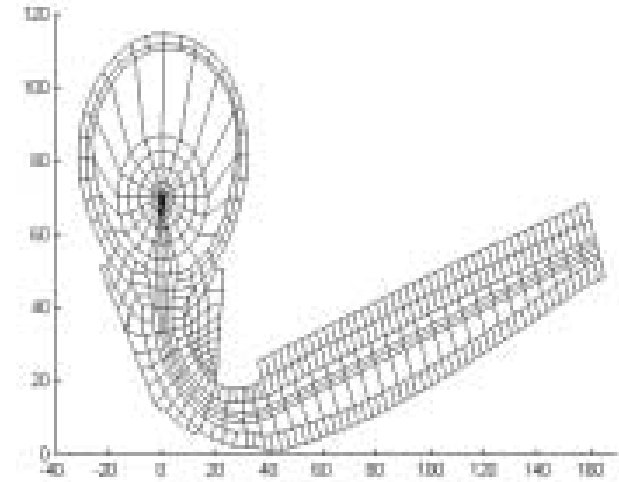

Pressure distribution in the urinary tract and velocity distribution in the bladder
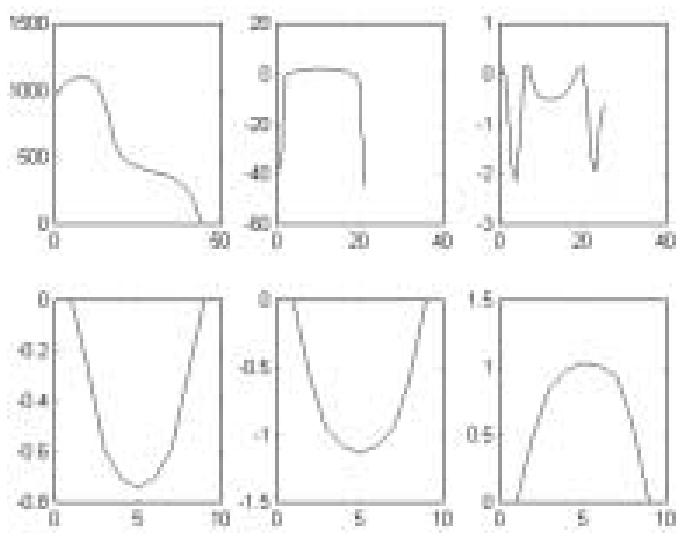

Velocity distribution in the urethra
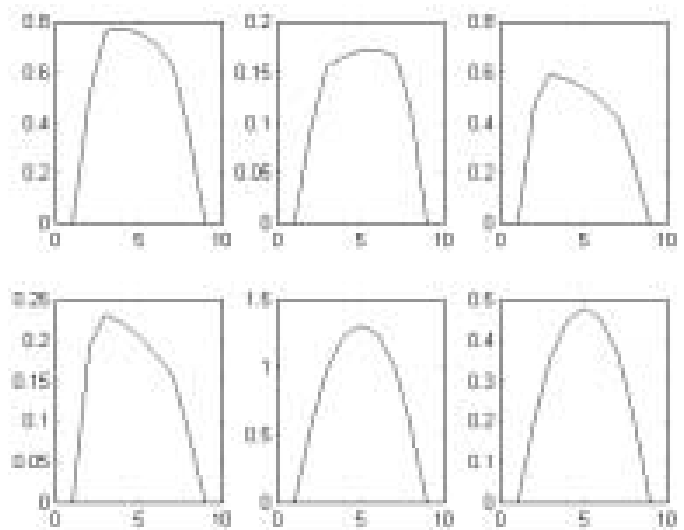

Fig. 3 Deformable model of the male urinary tract (plane model, flexible urethra) 

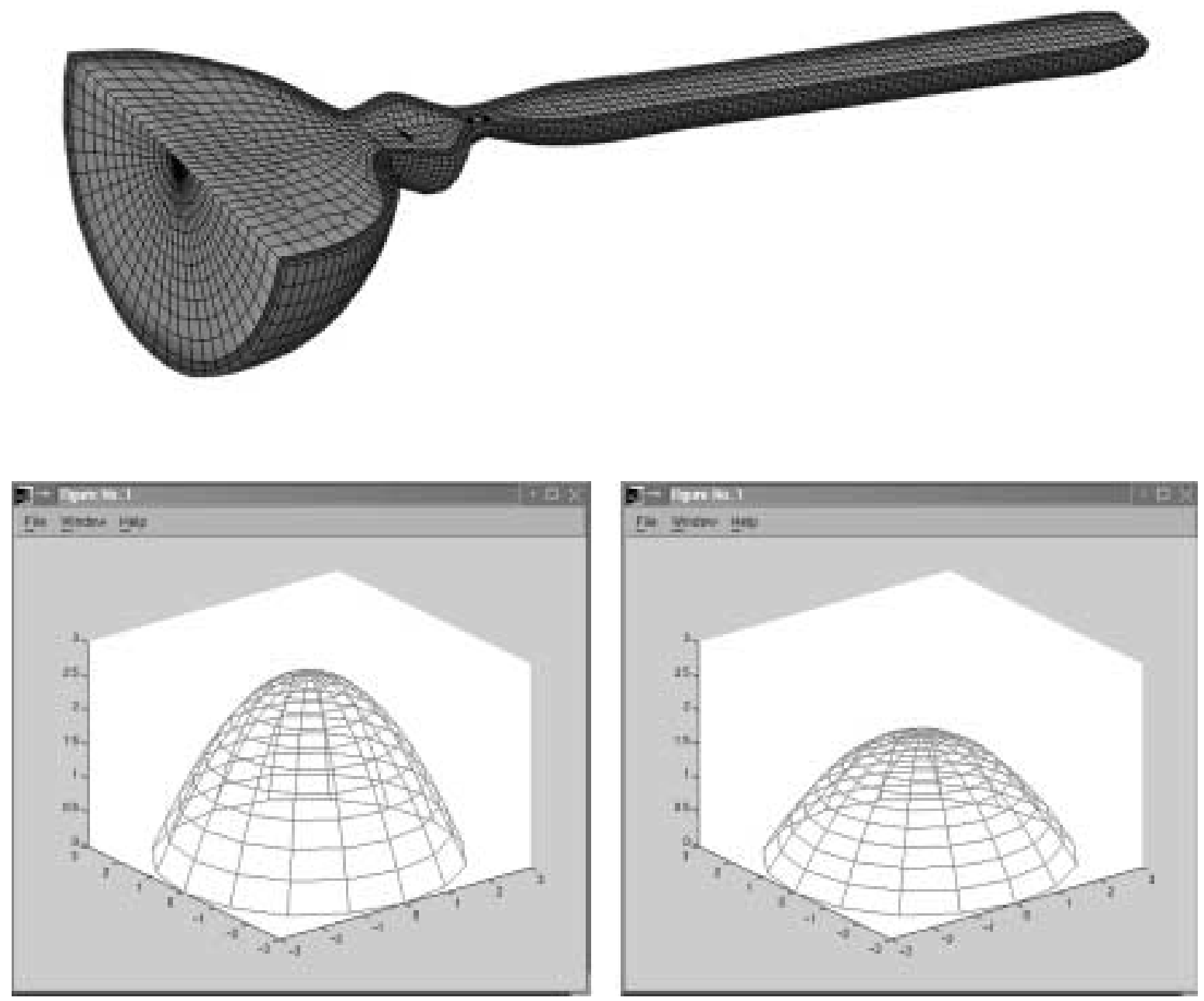

Fig. 4 Deformable model of the male urinary tract (3D model)

\section{ACKNOWLEDGEMENT}

This work was supported by grant of the GA $\check{C} R$, ,Modelling of tissues and organs in pelvic region with a special focus on the urinary tract" which is registered under the number 106/00/1733.

\section{REFERENCES}

1. Hughes, W. F., Brighton, J. A.: Theory and Problems of Fluid Dynamics. New York, McGRAW-HILL, INC. 1992.

2. Bathe, K. J.: Finite-Elemente- Methoden (Deutsche Übersetzung von P. Zimmermann). Berlin-Heidelberg, Springer-Verlag 1990.

3. Křen, J.: MKP v nelineární mechanice kontinua I. Výzkumná zpráva č. 102-09-97. Plzeň, ZČU, FAV, Katedra mechaniky 1997.

4. Křen, J.: Dynamics of the non-linear continuum. Zeszyty Naukowe, No. 11, Politechnika Slaska, Gliwice 1999.

5. Křen, J. a kol.: Biomechanika dolní části močového traktu. Závěrečná výzkumná zpráva grantu GA ČR č. 106/97/0397. Plzeň, ZČU, FAV, Katedra mechaniky 1999. 